\title{
A Randomized Controlled Trial and Radiographic Evaluation of Adjunctive Periodontal Treatment with Calcium and Vitamin D Supplementation
}

\author{
${ }^{1}$ Arpapan Bundit, ${ }^{2}$ Pusadee Yotnuengnit, ${ }^{3}$ Sirinun Wisetsin, ${ }^{4}$ Apichart Chittacharoen
}

\begin{abstract}
Aim: The objective of this study was to use clinical parameters, digital subtraction analysis, and computer intensity measurement to evaluate the effect of calcium and vitamin D supplementation in adult periodontitis patients with vertical bony defect after initial therapy.
\end{abstract}

Materials and methods: A total of 31 systemically healthy patients with moderate to severe chronic periodontitis and normal serum calcium level were included. The most obvious proximal vertical bony defect was selected in each patient as an area of interest (AOI). Subjects received initial periodontal therapy. They were randomized into two groups. The test group $(n=17)$ received calcium (600 mg twice daily) and vitamin $D(0.25 \mu \mathrm{g}$ once daily) supplements for 6 months. The control group $(n=14)$ received placebos. At baseline and 6 months, probing pocket depth (PPD), clinical attachment level (CAL), and standardized vertical bitewing radiographs were recorded for the AOI.

Results: A greater percentage of patients in the test group $(64.7 \%)$ than in the control group $(35.7 \%)$ gained bone according to both radiographic evaluation methods. At baseline and 6 months, no significant intragroup or intergroup differences in PPD and CAL were observed. Slightly greater PPD reduction and CAL gain were noted in the test group.

Conclusion: Periodontal healing of vertical bony defects was better among patients who used calcium and vitamin $D$ supplements than among control patients. Although the difference was not statistically different between the groups, the test group tended toward more improvement.

Keywords: Calcium and vitamin D supplements, Chronic periodontitis, Computer intensity measurement, Digital subtraction radiography, Vertical bony defect.

How to cite this article: Bundit A, Yotnuengnit $P$, Wisetsin $S$, Chittacharoen A. A Randomized Controlled Trial and Radiographic Evaluation of Adjunctive Periodontal Treatment with Calcium and Vitamin D Supplementation. Int J Experiment Dent Sci 2016;5(1):50-55.

Source of support: This study was supported by a grant from the Faculty of Dentistry, Mahidol University, and partially

\footnotetext{
${ }^{1}$ Student, ${ }^{2}$ Clinical Professor, ${ }^{3}$ Assistant Professor, ${ }^{4}$ Professor

${ }^{1-2}$ Department of Oral Medicine and Periodontology, Faculty of Dentistry, Mahidol University, Bangkok, Thailand

${ }^{3}$ Department of Oral Radiology, Faculty of Dentistry, Mahidol University, Bangkok, Thailand

${ }^{4}$ Department of Obstetrics and Gynecology, Faculty of Dentistry, Mahidol University, Bangkok, Thailand

Corresponding Author: Pusadee Yotnuengnit, Clinical Professor, Department of Oral Medicine and Periodontology Faculty of Dentistry, Mahidol University, Bangkok, Thailand Phone: 6622007841, e-mail: pussadee.yot@mahidol.ac.th
}

supported by the Graduate Studies Division of the Mahidol University Alumni Association.

Conflict of interest: None.

\section{INTRODUCTION}

Periodontitis is a destructive pathology of the periodontium, which results from the host's immunoinflammatory response to a bacterial challenge. Host modulation concept is raised to support periodontal tissue regeneration and reduce its destruction by downregulating destructive aspects of the host response and upregulating regenerative responses. ${ }^{1}$

Calcium is the most fundamental component of the human alveolar bone. Effective calcium intake is compounded of dietary content, absorption and retention efficiency, and various nutrient and drug interactions. The two determinants of effective calcium absorption are the amount of calcium in the diet and the efficiency of its absorption. ${ }^{2-4}$ The greatest absorption occurs when the dose is $<500 \mathrm{mg}$, taken twice daily after meals. Vitamin D intake improves calcium absorption.

Digital subtraction and computer intensity radiographs are methods used to define differences in bone changes before and after experiment. These techniques used computer-assisted imaging to convert different densities recorded on conventional X-ray film into digitized graylevel images. Using digital subtraction, the films of graylevel images after experiment are superimposed over the baseline films and the differences are subtracted. ${ }^{5}$

The objective of this study was to use clinical parameters and radiographic evaluation method to evaluate the effect of calcium and vitamin D supplementation as an adjunct treatment in adult periodontitis patients with vertical bony defect after initial therapy.

\section{MATERIALS AND METHODS}

\section{Case Selection}

This study was a double-blind, randomized controlled trial approved by the Committee for Human Research of Mahidol University; it was ethically conducted in accordance with the Declaration of Helsinki. Announcements 
were distributed to patients at the Faculty of Dentistry, Mahidol University, Bangkok, Thailand. The inclusion criteria was healthy patients older than 30 years of age and with at least one vertical proximal bone loss $\geq 2 \mathrm{~mm}$ in depth that was obvious on a radiograph. Those teeth had to be in use and occluded with an opposite natural tooth or prosthesis. Patients were excluded if they had uncontrolled systemic diseases, such as thyroid or parathyroid disease, abnormal absorption in the gastrointestinal tract; used to take calcium, vitamin D, estrogen, or progesterone hormones, antibiotics, or drugs that affect bone and calcium or vitamin $\mathrm{D}$ absorption; were smokers within the past 6 months; teeth with trauma from occlusion, or pulpal disease; had serum calcium levels higher than the upper normal limit. Patients who refused to continue or developed unwanted symptoms, allergy to either calcium or vitamin $\mathrm{D}$, were allowed to discontinue the study. All study participants had to provide written informed consent.

\section{Intraexaminer Error}

Calibration and reproducibility tests were performed by a dentist who was the only examiner by measured all parameters on ten patients who were not included in this study. Cohen's kappa was used to calculate the error.

Details of each visit as follows:

- The 63 patients with generalized moderate to severe chronic periodontitis (AAP 1999) and who had completed initial periodontal therapy were informed of the details of the study. A single tooth with obvious vertical bone loss was selected from their routine vertical bitewing radiographs. Thus, each patient's area of interest (AOI) was the single deepest site on one tooth.

- Those who provided written informed consent were instructed on oral hygiene practice, provided a soft toothbrush, and taught the modified Bass brushing and flossing techniques. They were asked to have blood tests to analyze their serum calcium level; only those who had calcium levels within the normal range $(6.7-10.0 \mathrm{mg} / \mathrm{dl})$ were included in the study.

- Patients were rescaling and re-root planing.

- (baseline visit) After 1 month, patients had to demonstrate adequate oral hygiene. All clinical parameters were examined for baseline data and all teeth were polished. Radiographs were retaken for the selected vertical proximal bone loss $\geq 2 \mathrm{~mm}$ in depth using the vertical bitewing technique. Individual acrylic stents were fixed with a bitewing holder. Randomization was performed using a table of random numbers to equally divide the test and control groups. Calcium carbonate supplementation tablets (600 mg: GPO ${ }^{\circledR}$ Bangkok, Thailand) and vitamin D capsules $\left(0.25 \mu \mathrm{g}\right.$ : CALCIT SG $\left.{ }^{\circledR}\right)$ were administered to the test group, while placebo tablets and capsules (Faculty of Pharmacology, Mahidol University, Bangkok, Thailand) were given to the control group. Both test and control groups took calcium supplements or placebos with meals twice daily and vitamin $\mathrm{D}$ or placebo capsules once daily. The examiner and the patients were blinded to the kind of supplement taken. Reminders to take the supplements were sent to all patients, periodically.

- At the second and fourth months after baseline, all patients were reexamined to evaluate gingival index (GI), plaque index (PI), and calculus index (CI), and all teeth were polished to remove supragingival plaque and remotivate oral hygiene. The supplements or placebos were provided to patients for 2 months.

- All parameters were reexamined at 6 months after the baseline visit.

\section{Digital Subtraction}

All radiographs at baseline and 6 months were evaluated for contrast discrepancies and position change. Differences in contrast were corrected by Adobe Photoshop. Positional discrepancies were adjusted using Image-Pro Plus version 7.0. A metal band was used to correct the density of structures in radiographs. Each pair of images was subtracted using Image-Pro Plus. Changes in the alveolar bone appearing in the subtracted image were interpreted in three categories as lighter (gain), unchanged, or darker (loss) areas, compared with the uniformly gray unchanged background. All comparisons were performed by agreement between two researchers under blinded conditions.

\section{Intensity Measurement}

The intensity of a pair of radiographs was measured using Image-Pro Plus version 7.0, and the intensity values were compared by matching areas 1 and 2 of each pair. Area 1, defined as the metal band, metal filling, or cusp tip of tooth, was created and assigned as a negative control, which was absolutely not involved with calcium metabolism. Area 2, at the selected defect site, was determined as an AOI that matched the outline of the vertical bony defects. All radiographic parameters were measured three separate times one day apart. The intraexaminer reproducibility was calibrated before the study. The different intensity value of area 2 was adjusted before use by subtracting it with the different intensity value of area 1 . 


\section{Statistical Analysis}

Mean probing pocket depth (PPD), clinical attachment level (CAL), and their changes at baseline and 6 months were calculated. Comparisons of PPD, CAL, CAL gain, $\mathrm{GI}, \mathrm{PI}$, and CI between test and control groups at different time intervals were performed using the Mann-Whitney U-test, for within groups the Wilcoxon signed-rank test; $\mathrm{p}<0.05$ was considered statistically significant. The percentages of patients that presented alveolar bone change, gain, unchanged, and loss, were calculated regarding radiographic evaluation method.

\section{RESULTS}

\section{Case Selection}

In all, 63 patients were interested, but only 38 patients met the inclusion criteria. They were asked to have a blood test, of which, three patients were excluded because they had serum calcium levels higher than the normal limit. The patients were divided by using a randomized table: Test group $(n=18)$ and control group $(n=17)$. Before the study began, two patients in the control group needed urgent treatment and one in the test group moved to another town. One month after baseline visit, one patient in the control group discontinued because of the inconvenience of taking supplements. Finally, 31 patients completed the study: Test group $(\mathrm{n}=17)$ and control group $(n=14)$. The demographic data are shown in Table 1.

Patients in both groups were healthy; however, five individuals in the test group and three in the control group had well-controlled hypertension. Smokers or

Table 1: Patient demographic data

\begin{tabular}{llll}
\hline & $\begin{array}{l}\text { All patients } \\
(n=31)\end{array}$ & $\begin{array}{l}\text { Test group } \\
(n=17)\end{array}$ & $\begin{array}{l}\text { Control group } \\
(n=14)\end{array}$ \\
\hline Mean age \pm SD & $50 \pm 10.49$ & $49 \pm 9.17$ & $51.21 \pm 12.14$ \\
(years) (range) & $(32-73)$ & $(32-63)$ & $(34-73)$ \\
Male & $6(19.35 \%)$ & $2(6.45 \%)$ & $4(12.90 \%)$ \\
Female & $25(80.65 \%)$ & $15(48.39 \%)$ & $10(32.26 \%)$ \\
\hline
\end{tabular}

$\mathrm{n}=$ number of patients diabetic patients were not found. None reported allergic or adverse effects until the end of study. Some patients noticed better general health, while others felt no difference. By counting supplements or placebos at every visit, both groups were observed to take the supplements consistently.

\section{Clinical Measurement}

Intraexaminer error was summarized for CI (0.984), PPD (0.993), and cementoenamel junction to gingival margin (0.991). The Mann-Whitney U-test indicated that the mean PI and GI scores at baseline and months 2, 4, and 6 did not differ significantly between the test and control groups. Among within-groups, Wilcoxon signed rank test indicated that the mean PI and GI scores differed significantly at baseline and at months 4 and 6 (Table 2) .

There was no statistically significant difference in the mean CI score between test and control groups at months 2 and 4 , but significant difference $(p=0.00)$ in months 6 . Statistically significant differences in mean CI score were apparent within group at every time point in both groups respectively $(p=0.01,0.00$, and 0.00 in the test group and $\mathrm{p}=0.03,0.01$, and 0.01 in the control group at months 2 , 4 , and 6 (Table 2).

At baseline, the PPD range was $4-10 \mathrm{~mm}$. No statistically significant difference was observed between the test and control groups at baseline or at 6 months. Regarding PPD reduction, a CAL gain was observed in both treatment groups (52.94\% in the test group, $42.86 \%$ in the control group). However, no statistically significant differences in CAL or CAL gain were noted (Table 3).

\section{Radiographic Evaluation}

Regarding intensity measurement, the mean AOI intensity values were adjusted by the mean negative control value (Fig. 1). The calibrated AOI intensity values were interpreted as gain and loss when compared with baseline values (Table 4). Gains were noted in 64.7 and $35.7 \%$ of patients in the test and control groups respectively. Losses were noted in 35.2 and $64.2 \%$ of patients in the test and

Table 2: $\mathrm{PI}, \mathrm{Gl}$, and $\mathrm{Cl}$ scores (mean $\pm \mathrm{SD}$ ) in test and control groups at baseline and months 2, 4, and 6

\begin{tabular}{|c|c|c|c|c|c|}
\hline \multirow[b]{2}{*}{ Index } & \multirow[b]{2}{*}{ Patient group } & \multicolumn{4}{|c|}{ Mean $\pm S D$} \\
\hline & & Baseline & 2 months & 4 months & 6 months \\
\hline \multirow[t]{2}{*}{$\mathrm{PI}$} & Test & $0.37 \pm 0.15$ & $0.39 \pm 0.16$ & $0.54 \pm 0.15^{\star}$ & $0.61 \pm 0.18^{*}$ \\
\hline & Control & $0.42 \pm 0.15$ & $0.51 \pm 0.20$ & $0.54 \pm 0.16^{*}$ & $0.64 \pm 0.11^{*}$ \\
\hline \multirow[t]{2}{*}{$\mathrm{GI}$} & Test & $0.19 \pm 0.17$ & $0.21 \pm 0.14$ & $0.35 \pm 0.15^{*}$ & $0.44 \pm 0.12^{*}$ \\
\hline & Control & $0.20 \pm 0.14$ & $0.26 \pm 0.15$ & $0.34 \pm 0.10^{*}$ & $0.48 \pm 0.17^{*}$ \\
\hline \multirow[t]{2}{*}{$\mathrm{Cl}$} & Test & $0 \pm 0.00$ & $0.127 \pm 0.98^{*}$ & $0.30 \pm 0.11^{*}$ & $0.56 \pm 0.09^{*}$ \\
\hline & Control & $0.02 \pm 0.07$ & $0.196 \pm 0.11^{*}$ & $0.33 \pm 0.10^{*}$ & $0.34 \pm 0.08^{*, \star *}$ \\
\hline
\end{tabular}

*Statistically significant difference of mean when compared with the baseline; ** Statistically significant difference of mean when compared between test and control groups; PI: plaque index; GI: gingival index; Cl: calculus index; SD: standard deviation 

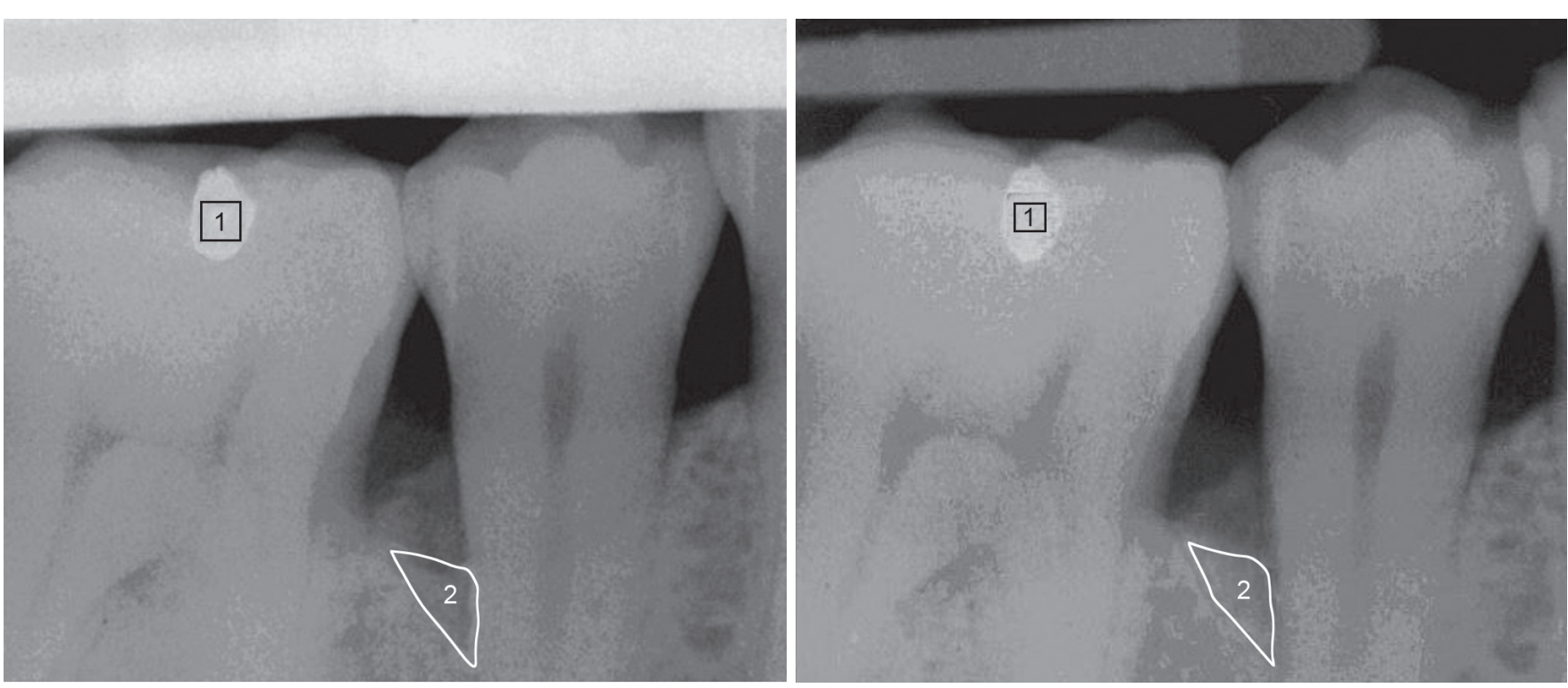

Fig. 1: The areas of interest (2) and negative control areas (1) were determined and created. The intensity of each area was measured using the Image-Pro Plus program

Table 3: Probing pocket depth (PPD) and clinical attachment level $(C A L)($ mean $\pm S D)$ at baseline and at 6 months

\begin{tabular}{lllll}
\hline & & \multicolumn{3}{c}{ Mean $\pm S D(\mathrm{~mm})$} \\
\cline { 3 - 5 } & Patient & & & PPD reduction/ \\
& group & Baseline & 6 months & $C A L$ gain \\
\hline PPD & Test & $6.82 \pm 1.55$ & $6.18 \pm 2.04$ & $0.65 \pm 1.06$ \\
& Control & $6.14 \pm 1.03$ & $5.50 \pm 1.29$ & $0.64 \pm 0.84$ \\
CAL & Test & $6.41 \pm 2.03$ & $5.94 \pm 2.36$ & $0.47 \pm 1.18$ \\
& Control & $5.79 \pm 1.19$ & $5.50 \pm 1.70$ & $0.36 \pm 1.01$ \\
\hline
\end{tabular}

SD: standard deviation

Table 4: Comparison of pairs of radiographs in areas of interest using computer intensity radiography

\begin{tabular}{lll}
\hline & \multicolumn{2}{l}{ Computer intensity radiography } \\
\cline { 2 - 3 } Patient group & Gain $(n / \%)$ & Loss $(n / \%)$ \\
\hline Test $(n=17)$ & $11(64.7 \%)$ & $6(35.2 \%)$ \\
Control $(n=14)$ & $5(35.7 \%)$ & $9(64.2 \%)$ \\
\hline
\end{tabular}

Table 5: Comparison of pairs of radiographs using digital subtraction analysis

\begin{tabular}{llll}
\hline & \multicolumn{3}{c}{ Digital subtraction analysis } \\
\cline { 2 - 4 } Patient group & Gain & No change & Loss \\
\hline Test $(\mathrm{n}=17)$ & $11(64.7 \%)$ & $2(11.7 \%)$ & $4(23.5 \%)$ \\
Control $(\mathrm{n}=14)$ & $5(35.7 \%)$ & $4(28.5 \%)$ & $5(35.7 \%)$ \\
\hline
\end{tabular}

control groups respectively. Using Pearson's Chi-squared test, no statistically significant difference was observed between test and control groups.

For digital subtraction analysis, the radiographs were interpreted with respect to three categories (gain, no change, and loss; Table 5). Gains were noted in 64.7\% of patients in the test group and $35.7 \%$ of patients in the control group. No change was noted in 11.7 and $28.5 \%$ of patients in the test and control groups respectively. Losses were noted in 23.5 and $35.7 \%$ of patients in the test and control groups respectively.

\section{DISCUSSION}

This randomized controlled clinical study aimed to evaluate the outcomes of initial periodontal treatment enhanced by oral calcium and vitamin D supplementation as adjunctive treatment for 6 months. Vertical bone defect was selected for use because it has the potential to regenerate the lost periodontium. It is possible that calcium and vitamin D supplementation may provide positive effects and yield better results than conventional treatment. ${ }^{6,7}$ However, responses to treatment type varied between individual patients. A number of factors have been suggested to play a role in healing after periodontal treatment. ${ }^{8}$ In this study, the mean CAL showed more gain in the test group than in the control group. Regarding the radiographic parameter, the patients in the test group exhibited a higher percentage of gain than the control group in the intensity measurement and in digital subtraction analysis, although the differences were not statistically significant. These results might indicate that calcium and vitamin D supplementation helps support the healing of intrabony defects after nonsurgical treatment.

However, radiographs are two-dimensional images. The gain in intensity might reflect the increasing intensity from the surrounding bony walls with or without a real increase of intensity within the AOI. ${ }^{9}$ Gains, therefore, in the control group were seen.Smoking, which is the factor that could affect bone healing, were excluded from this study. That could be the explanation for some positive outcomes to the usual initial phase of periodontal therapy, especially within vertical intrabony defects. ${ }^{10}$ 
The trend of better results observed in the test group regarding CAL and radiographic parameters might have been affected by calcium and vitamin D supplementation. A previous study reported that calcium and vitamin D are centrally involved in bone metabolism and homeostasis. ${ }^{11}$ Most chronic periodontitis patients were nearly middleaged to elderly individuals. The effect of calcium absorption depends on the amount of calcium in the diet and the efficiency of its absorption. Calcium absorption is mediated by vitamin D and effectively declines after middle age. The test group received calcium carbonate $(1,200 \mathrm{mg} /$ day $)$ containing approximately $480 \mathrm{mg}$ pure calcium per day, which does not reach the toxic calcium dose of 2,500 mg/day. ${ }^{12}$ For vitamin D, the test group took $0.25 \mu \mathrm{g}(10 \mathrm{IU})$ per day, far from the toxic dose of $10 \mu \mathrm{g}$ (400 IU) per day. ${ }^{13}$ In this study, a significant difference in CI scores between the test and control groups at 6 months was observed. Increased CI scores in the test group may be explained by calcium metabolism. This finding may be attributed to the calcium ion concentration in gingival crevicular fluid and saliva. Calcium ions are secreted to maintain calcium homeostasis in several processes. Secreting calcium ions through saliva is another method of maintaining calcium ion homeostasis. ${ }^{14}$

After initial treatment, PI, GI, and CI scores increased in both groups as time passed, and significant differences were observed in the intragroup at months 4 and 6. Regenerated intrabony defects that were treated nonsurgically had less bone healing when inadequate oral hygiene was established. Even though calcium and vitamin D have positive effects on bone healing, they could not be accounted responsible for the entire healing process. Therefore, losses can occur in both the test and control groups.

All radiographs were taken by the same researcher with the same dental X-ray machine and customized acrylic stent to control the position and angulation quality. An automatic film-processing machine with fresh developer and fixer solution was used to process all films.

Digital subtraction and computer intensity measurements were employed to extract additional information from the radiographs that cannot be visualized. By digital subtraction, the pair of radiographs compared were strictly controlled regarding the condition described; otherwise, it was difficult to interpret. ${ }^{5,15}$

Computer intensity measurement was able to confirm the results of digital subtraction. The original image was directly measured. Two areas in the radiographs determined the negative control and the AOI. Although the radiographs were controlled at every step of production, different intensities of the negative control were still observed. The AOI and negative control of every image were measured. The different intensities of AOIs were subtracted with the different intensity of the negative control for the final calibration of the different intensities of the AOI. With the help of a stent, meticulous film processing, and calibrated values, random errors in positioning periapical radiographs and image processing were minimized in this study.

The results of computer intensity and digital subtraction analyses were identical with respect to gains and losses. When the pattern of gain or loss was not homogeneous, these areas were grouped as unchanged in digital subtraction analysis. Computer intensity measurement was able to detect more delicately than the subtraction analysis. Then, unchanged group according to the digital subtraction technique exhibited losses by computer intensity.

\section{CONCLUSION}

Periodontitis is inflammation of the tissues surrounding the tooth affecting the gingiva, periodontal ligaments and the bone and in its severe forms there can be loss of bone that supports the tooth, resulting in the tooth becoming loose and even causing tooth loss. ${ }^{16}$ Microorganisms in dental plaque interact with neutrophils/monocytes resulting in acute-phase inflammatory response by the systemic dissemination of locally produced mediators ${ }^{17}$ which lead to localized destruction of bone supporting structure.

The result of this controlled clinical study could be concluded that periodontal healing in patients with vertical bone defects is better for those who take calcium and vitamin D supplements than for those who do not. Although no statistically significant differences between the test and control were observed, the test group tended to exhibit greater improvement. A longer study term and a larger sample size should be considered in future study.

\section{REFERENCES}

1. Korman KS. Host modulation as a therapeutic strategy in the treatment of periodontal disease. Clin Infect Dis 1999 Mar;28(3):520-526.

2. Alevizaki CC, Ikkos DC, Singhelakis PJ. Progressive decrease of true intestinal calcium absorption with age in normal man. Nucl Med 1973 Oct;14(10):760-762.

3. Nordin BEC, Williams R, Marshall DH, Gallagher JC, Williams A, Peacock M. Calcium absorption in the elderly. Calcif Tissue Res 1976 Aug;2l(Suppl):442-451.

4. Ireland P, Fordtran IS. Effect of dietary calcium and age on jejunal calcium absorption in humans studied by intestinal perfusion. J Clin Invest 1973 Nov;52(11):2672-2681.

5. Jeffcoat MK, Reddy MS, Magnusson I, Johnson B, Meredith MP, Cavanaugh PF Jr, Gerlach RW. Efficacy of quantitative 
digital subtraction radiography using radiographs exposed in a multicenter trial. J Periodontal Res 1996 Apr;31(3): 157-160.

6. Hildebolt CF. Effect of vitamin D and calcium on periodontitis. J Periodontol 2005 Sep;76(9):1576-1587.

7. Garcia M, Hildebolt CF, Miley DD, Dixon DA, Couture RA, Spearie CL, Langenwalter EM, Shannon WD, Deych E, Mueller C, et al. One-year effects of vitamin D and calcium supplementation on chronic periodontitis. J Periodontol 2011 Jan;82(1):25-32.

8. Claffey N, Polyzois I, Ziaka P. An overview of nonsurgical and surgical therapy. Periodontol 20002004 Oct;36(1): 35-44.

9. Christgau M, Hiller KA. Quantitative digital subtraction radiography for the determination of small changes in bone thickness. Oral Surg Oral Med Oral Pathol Oral Radiol Endod 1998 Apr; 85(4):462-472.

10. Lang NP. Focus on intrabony defects-conservative therapy. Periodontol 20002000 Feb;22(1):51-58.

11. Heaney RP, Gallagher JC, Johnston CC, Neer R, Parfitt AM, Whedon GD. Calcium nutrition and bone health in the elderly. Am J Clin Nutr 1982 Nov;36(5 Suppl):986-1013.
12. Standing Committee on the Scientific Evaluation of Dietary Reference Intakes, Food and Nutrition Board, Institute of Medicine. Dietary Reference Intakes for Calcium, Phosphorus, Magnesium, Vitamin D and Fluoride. Washington, DC: National Academy Press; 1997.

13. Chesney RW. Vitamin D: can an upper limit be defined? J Nutr 1989 Dec;119(12 Suppl):1825-1828.

14. Anerud A, Löe H, Boysen $H$. The natural history and clinical course of calculus formation in man. J Clin Periodontol 1991 Mar;18(3):160-170.

15. Hausmann E, Allen K, Loza J, Buchanan W, Cavanaugh PF Jr. Validation of quantitative digital subtraction radiography using the electronically guided alignment device/ impression technique. J Periodontol 1996 Sep;67(9):895-899.

16. Saini R. A prospective experimental comparative study on the clinical effects of calculus dissolution based oral rinse in gingivitis patients. Int J Experiment Dent Sci 2015;4(1): 33-39.

17. Saini R. Study on C-reactive Protein in Periodontal Diseases: Estimation and Correlation in Health, Gingivitis and Periodontitis and its Assessment after Scaling and Root Planing. Int J Experiment Dent Sci 2014;3(1):4-7. 